\title{
ÉTUDES DE FILTRES ISOTOPIQUES POUR LES MASERS AU RUBIDIUM
}

\author{
G. MISSOUT, R. VAILLANCOURT, M. TÊTU et J. VANIER \\ C. R. A. M. Laboratoire d'Electronique Quantique, Département de Génie Electrique \\ Université Laval, Québec 10, P. Québec, Canada
}

(Reçu le 24 février 1971)

\begin{abstract}
Résumé. - Le filtrage isotopique d'une lampe au rubidium 85 , à l'aide de l'isotope 87 , a été étudié ; en particulier, on a fait ressortir l'influence sur le filtrage de la nature et de la pression du gaz tampon contenu dans le filtre. On montre en outre qu'il est possible de réaliser un filtrage efficace en utilisant de l'hélium comme gaz tampon. Ces études ont été faites à l'aide d'un spectromètre FabryPérot dont une description sommaire est faite. Ces études ont de plus permis la réalisation d'un maser $\mathrm{Rb} 85$.
\end{abstract}

\begin{abstract}
The filtering of a rubidium 85 lamp by a filter containing rubidium 87 has been studied ; the influence of the nature and of the pressure of the buffer gas on the efficiency of the filter is shown. It is shown in particular that helium used as a buffer gas makes possible the construction of efficient filters. A short description of the Fabry-Pérot interferometer used in these studies is given. These studies have made possible the realization of a $\mathrm{Rb} 85$ maser.
\end{abstract}

Introduction. - Le pompage optique du type hyperfin est couramment employé dans les horloges atomiques passives utilisant la fréquence hyperfine de l'atome de rubidium 87 comme référence [1]. Ce type de pompage permet d'obtenir une inversion de population très prononcée entre les niveaux hyperfins de l'état fondamental de cet atome, rendant possible la mise en évidence de transitions entre ces mêmes niveaux. D'autre part, ce même type de pompage a aussi permis la réalisation de l'oscillateur atomique au rubidium 87 [2].

Ces standards de fréquence, basés sur la fréquence de transition hyperfine du rubidium, jouent actuellement un grand rôle dans les domaines qui demandent des appareils de faibles dimensions et de grande stabilité de fréquence. Présentement l'horloge passive au rubidium 87 possède une stabilité de fréquence à long terme, relativement bonne [3]. Toutefois la stabilité à court terme dépend de la stabilité du pilote au quartz qui est asservie à la raie de résonance atomique. L'étendue de la région où la stabilité de l'appareil est fixée par le pilote est fonction du rapport signal-bruit du signal de résonance et de la constante de temps d'asservissement. Dans ce domaine, le maser à rubidium 87 semble présenter de nets avantages [4], [5]. En effet, sa grande puissance de sortie permet d'avoir un rapport signal-bruit plus avantageux. D'autre part, le fait qu'il soit lui-même un oscillateur donne plus de liberté sur le choix de la constante de temps d'asservissement, permettant même de se passer de pilote pour certaines applications.

D'un autre côté, des calculs simples montrent que la puissance du maser à rubidium 85 peut être supérieure à celle du maser au rubidium 87 conduisant du même coup à une stabilité à court terme plus grande. Toutefois, le pompage optique hyperfin de ce dernier maser n'est pas aussi facile que dans le cas du rubidium 87 ; ceci est dû à la proximité des raies hyperfines du rubidium 85 , en comparaison de celles du rubidium 87 . Certains résultats partiels ont déjà été publiés en relation avec le pompage hyperfin de l'isotope 85 du rubidium [6], [7]. Cependant, afin d'avoir une meilleure compréhension du filtrage isotopique sous différentes conditions (gaz tampons, température...), nous avons fait une étude plus approfondie des lampes et filtres utilisés dans le maser au rubidium 85. Dans cette publication on décrit d'abord le principe du pompage hyperfin. Ensuite on fait ressortir l'importance jouée par la grandeur du taux de pompage dans la réalisation des masers au rubidium. Enfin on décrit le montage avec lequel on a étudié divers filtres et lampes au rubidium et on donne les résultats expérimentaux.

I. Le pompage optique. - Considérons trois niveaux notés 1,2 et 3 , classés par ordre croissant d'énergie, tels que représentés sur la figure 1 .

Dans les cas qui nous intéressent, les niveaux 1 et 2 sont les niveaux hyperfins de l'état fondamental et la fréquence de la transition correspondante est de l'ordre de quelques gigahertz. L'état 3 est un état excité ; la longueur d'onde correspondant à la transition $(3) \leftrightarrow(2,1)$ est de l'ordre de $8000 \AA\left(10^{15} \mathrm{~Hz}\right)$. Aux températures utilisées dans nos expériences, seuls les niveaux 1 et 2 sont peuplés; de plus, à l'équilibre, en l'absence de perturbations externes, ces deux niveaux ont sensiblement la même population.

Le cycle de pompage optique peut être décrit comme suit. Un photon d'énergie appropriée excite un atome du niveau 1 au niveau 3. L'atome peut alors 


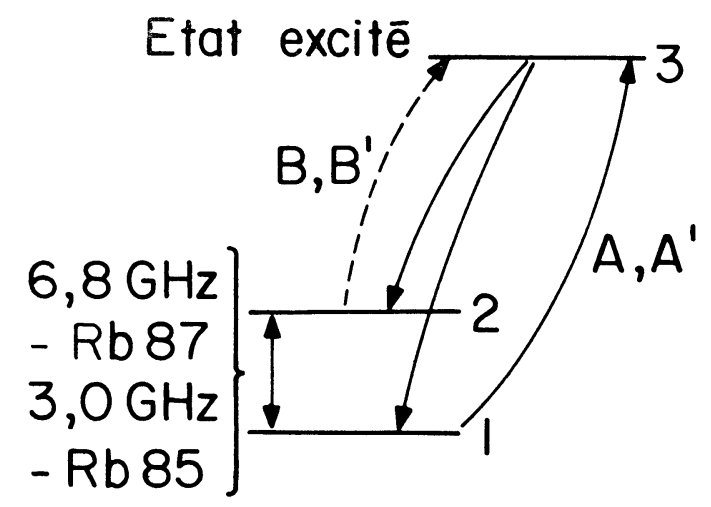

FIG. 1. - Schéma simplifié des niveaux d'énergie du rubidium intervenant dans le pompage optique.

retomber dans l'état fondamental par un procédé d'émission spontanée ou encore par un procédé non radiatif tel que celui qui intervient lors de collisions non élastiques entre l'atome en question et un atome de gaz tampon tel que l'azote. Ce dernier cas est celui qui nous intéresse particulièrement; on fait alors l'hypothèse que la retombée de l'atome vers les niveaux 1 et 2 est équiprobable. Ce processus global, appelé cycle de pompage optique, appliqué à un ensemble d'atomes, peut produire des différences de populations sensibles entre les niveaux 2 et 1 , si les transitions produites entre ces deux niveaux par la relaxation sont peu nombreuses relativement à celles produites par le pompage optique.

En général, il est difficile d'obtenir un spectre de raies optiques avec les caractéristiques propres à ne produire des transitions que du niveau 1 au niveau 3, tout en n'affectant pas le niveau 2. Toutefois, dans le cas des isotopes 85 et $87 \mathrm{du}$ rubidium, il existe une coïncidence heureuse entre les raies correspondant à des transitions du niveau 2 au niveau 3 . Une lampe à décharge au rubidium 87 , par exemple, aura sa composante nuisible $(2 \leftrightarrow 3)$ absorbée par un filtre contenant du rubidium 85 et réciproquement. Le pompage sélectif décrit plus haut est donc possible avec le rubidium.

II. Les lampes au rubidium et leur filtrage. Dans le rubidium, l'état fondamental est un état ${ }^{2} \mathrm{~S}_{1 / 2}$. Cet état est formé de deux niveaux hyperfins avec moment angulaire $F=1$ et $F=2$ pour le rubidium $87, F=2$ et $F=3$ pour le rubidium 85 . Le pompage a lieu entre l'état ${ }^{2} S_{1 / 2}$ et les états $P_{1 / 2}$ et $P_{2 / 3}$. Les raies $D_{1}$ et $D_{2}$ liant ces niveaux ont pour longueur d'onde respective $7947 \AA$ et $7800 \AA$. Les énergies de multiplet ${ }^{2} S_{1 / 2}$ ont été mesurées par $B$. Bederson et V. Jaccarino [8], celles des multiplets ${ }^{2} \mathrm{P}_{1 / 2}$ et ${ }^{2} \mathrm{P}_{3 / 2}$ par B. Senitzky et I. I. Rabi [9].

Le tableau I donne la position et l'énergie des raies de la structure hyperfine du rubidium naturel. Ces raies sont repérées par rapport au centre de la structure fine et leurs intensités sont normalisées pour que la plus grande soit égale à 100 . Ces raies ne sont pas complètement résolues, dues à l'effet Doppler qui crée un élargissement de l'ordre de $500 \mathrm{MHz}$ aux températures utilisées.

\section{TABleau I}

Intensités et position de raies de la structure hyperfine du rubidium naturel. Les raies dues aux deux isotopes sont notées séparément (d'après Gibbs [10])

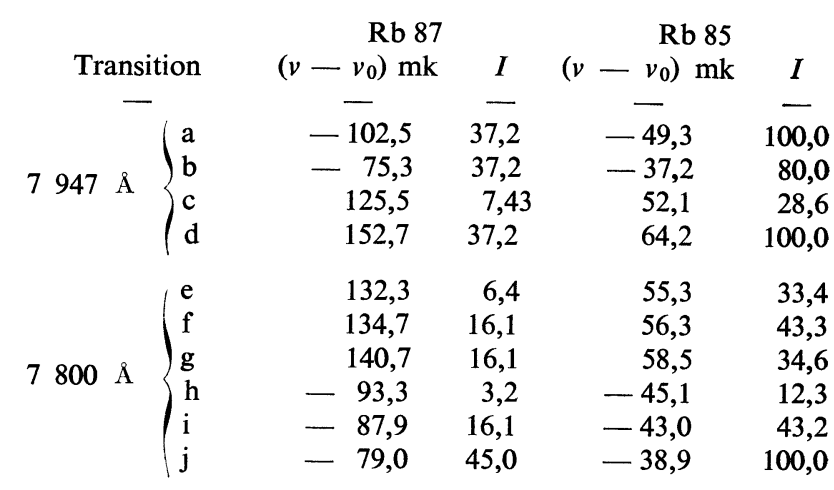

En pratique, on utilise une lampe contenant quelques milligrammes de l'isotope approprié du rubidium et un gaz d'amorce (Krypton) sous une pression de quelques torrs. La lampe est excitée par un oscillateur radiofréquence. Le filtre placé devant la lampe contient de son côté quelques milligrammes de l'isotope non utilisé dans la lampe. Pour rendre le filtrage optimum, on introduit dans le filtre un gaz tampon dont la fonction est de déplacer le groupe de raies d'absorption dans un sens ou dans l'autre, améliorant ainsi la coïncidence avec la raie à absorber; en même temps, on écarte les raies d'absorption de la raie utile.

Dans le cas du rubidium 85, il faut déplacer les raies d'absorption du filtre vers le violet. Dans celui du rubidium 87 , il faut les déplacer vers le rouge. Les études de A. Ch'en [11] nous ont guidés dans le choix des gaz tampons.

L'équation d'absorption dans le filtre [12] à la forme suivante : $I=I_{0} \exp \left(-k_{v} x\right)$, où $k_{v}$ est une constante qui dépend linéairement de la densité de rubidium. A une température de $80^{\circ} \mathrm{C}$, l'épaisseur théorique nécessaire pour réduire à la sortie du filtre l'intensité à $1 / \mathrm{e}$ de sa valeur à l'entrée du filtre est voisine de $0,7 \mathrm{~mm}$. En fait, il y a pompage optique du filtre et celui-ci devient transparent. Expérimentalement, on fixe l'épaisseur du filtre au voisinage de $25 \mathrm{~mm}$. Le rubidium est placé en excès dans le filtre et la température de celui-ci fixe la tension de vapeur du rubidium.

III. Les masers au rubidium. - Des théories décrivant approximativement le comportement du maser au rubidium 87 ont été publiées par J. Vanier [5] et E. I. Alekseyev et N. Bazarov [13]. L'équation donnant les conditions d'oscillations du maser au rubidium 85 a été donnée dans la référence [5]. Ces 
théories sont développées dans les cadres de la théorie générale du pompage optique donnée par Barrat et Cohen-Tannoudji [14] et dans l'hypothèse du pompage homogène de la cellule $\left({ }^{1}\right)$. Les résultats expérimentaux obtenus [5], [15] sur le maser au rubidium 87 sont en accord qualitatif avec la théorie et il est montré que le taux de pompage optique est un paramètre très critique dont dépend le comportement du maser. En particulier, on montre qu'à une température donnée du maser, il existe des valeurs de taux de pompage en dessous et au-dessus desquelles les oscillations ne sont pas permises. A des températures élevées du maser où la tension de vapeur du rubidium est grande, le pompage devient difficile et en pratique le problème est d'obtenir un taux de pompage suffisant. Dans toutes les expériences rapportées, la puissance permise est limitée par le taux de pompage.

IV. Le spectromètre. - Nous avons choisi un Fabry-Pérot à cause de sa simplicité de réalisation et de sa bonne adaptation à notre problème. Les caractéristiques de ce F. P. ont été calculées en partant des résultats de Chabbal [16]. Le diamètre utile des miroirs est de $63,5 \mathrm{~mm}$. La précision de surface des miroirs est de $\lambda / 200$ à la raie verte du mercure. Les miroirs sont recouverts d'une couche réflectrice créée par un empilement de diélectrique. La largeur de bande est de $2000 \AA$. Elle est centrée sur $7900 \AA$. Un pic de réflexion à $6328 \AA$ permet d'utiliser un laser à hélium-néon pour centrer le système. Chaque miroir laisse passer $5 \%$ de lumière incidente aux longueurs d'ondes qui nous intéressent. Le balayage du Fabry-Pérot se fait de façon pneumatique; les miroirs sont enfermés dans une enceinte en aluminium fermée par deux fenêtres aux faces planes à $\lambda / 4$. Le vide est fait dans cette enceinte, puis l'air atmosphérique desséché y pénètre par une fuite supersonique (tube capillaire) à débit constant. La linéarité de ce balayage est excellente sur les cinq premiers modes. Pour éviter les réflexions parasites, les deux faces de chaque pièce de verre font entre elles un angle de $30^{\prime}$. La lentille utilisée est achromatique et permet un réglage en lumière visible; sa longueur focale est de $300 \mathrm{~mm}$. Une paire de filtres interférentiels permet de choisir un groupe de raies (soit $D_{1}$, soit $D_{2}$ ) dans le but d'éviter les recouvrements d'ordres. La largeur de bande de ces filtres est de $100 \AA$ et la transmission est $75 \%$ et $50 \%$ de la lumière incidente pour les raies $D_{1}$ et $D_{2}$ respectivement. Le système détecteur est composé d'un écran percé d'un trou de $0,33 \mathrm{~mm}$ et d'un photomultiplicateur RCA 7102 refroidi à l'azote liquide.

(1) Des calculs récents sur un ordinateur ont toutefois montré que l'inhomogénéité du pompage joue un rôle très important dans la valeur absolue de la puissance de sortie du maser. Cependant l'allure des courbes de puissance en fonction du taux de pompage est à peu près la même que celle obtenue par la théorie approximative décrite dans la référence [5].
V. Résultats. - La figure 2 illustre la forme du spectre $\mathrm{D}_{1}$ obtenu dans le cas d'une lampe au rubidium naturel. Cette figure donne une bonne idée de la largeur des raies en fonction de leur espacement.

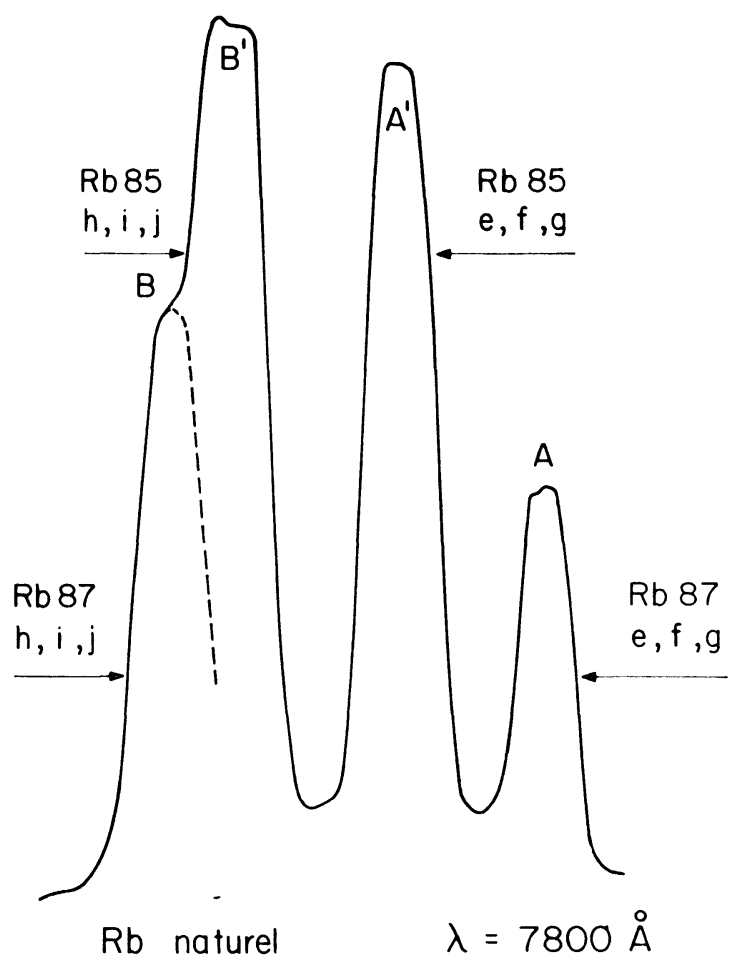

FIG. 2. - Spectre d'une lampe au rubidium naturel obtenu avec le montage décrit dans le texte. La contribution de chaque isotope est indiquée. La raie $\mathrm{D}_{2}$ seulement est représentée ici.

a) Filtrage D'Une lampe au Rubidium 87. Cette étude n'a été faite qu'à titre comparatif, l'optimisation du filtrage ayant déjà été réalisée par d'autres voies. La lampe contient du rubidium 87 et 2 torrs de krypton servant à amorcer la décharge ; elle est maintenue au voisinage de $115^{\circ} \mathrm{C}$. Le filtre contient du rubidium 85 et 50 torrs d'argon. La figure 3 nous

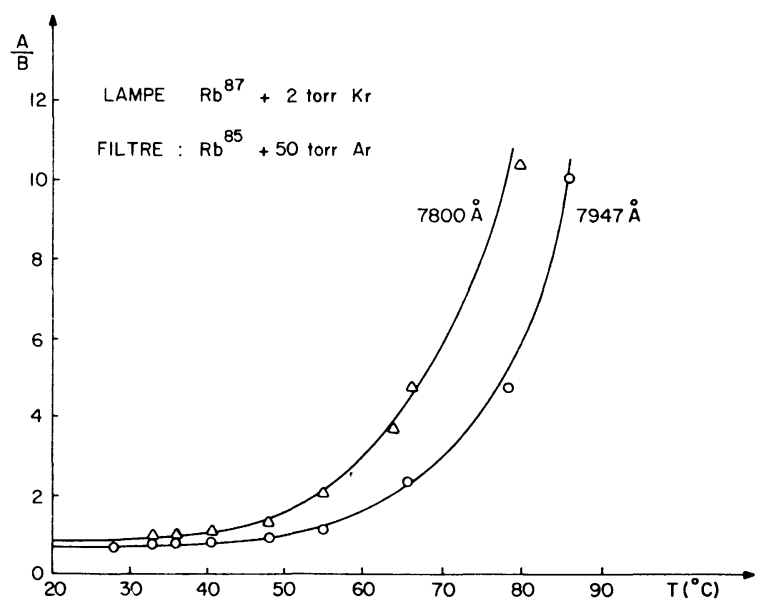

Fig. 3. - Influence de la température d'un filtre isotopique au rubidium 85 sur le rapport des amplitudes des composantes des deux doublets $\left(D_{1}\right.$ et $\left.D_{2}\right)$ du spectre hyperfin d'une lampe au rubidium 87 . 
montre le rapport des raies $\mathrm{A}$ et $\mathrm{B}$ en fonction de la température du filtre, pour $\lambda=7800 \AA$ et $\lambda=7947 \AA$ $\left({ }^{2}\right)$.

b) Filtrage D'une LAmpe au Rubidium 85. La lampe contient du rubidium 85 et 2 torrs d'argon; elle est maintenue à $110^{\circ} \mathrm{C}$. Le filtre contenant du rubidium 87 est branché sur une machine pneumatique permettant de faire varier la nature et la pression du gaz tampon. Les deux raies $D_{1}$ et $D_{2}$ sont examinées. Les courbes des figures 4 et 5 nous donnent le rapport

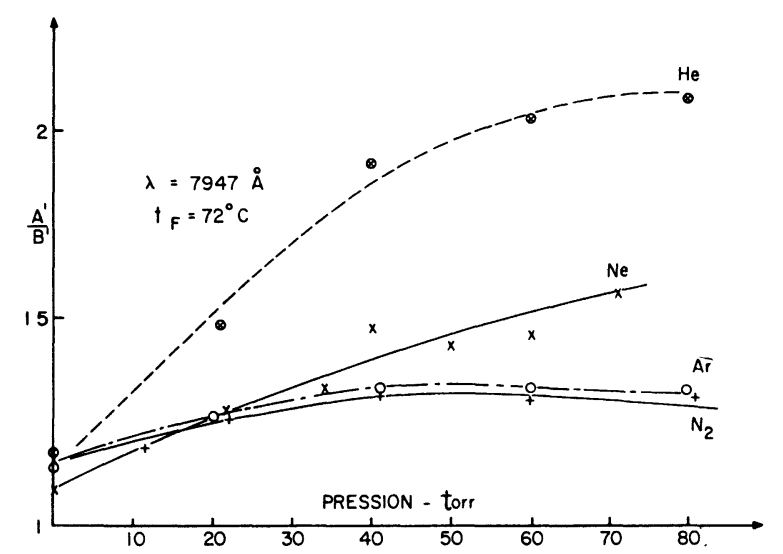

FIG. 4. - Filtrage d'une lampe au rubidium 85 par un fitre isotopique au rubidium 87 . On montre l'influence de la pression et de la nature du gaz tampon contenu dans le filtre, sur le rapport $A^{\prime} / B^{\prime}$. Les résultats donnés sont pour la raie $\mathrm{D}_{1}$.

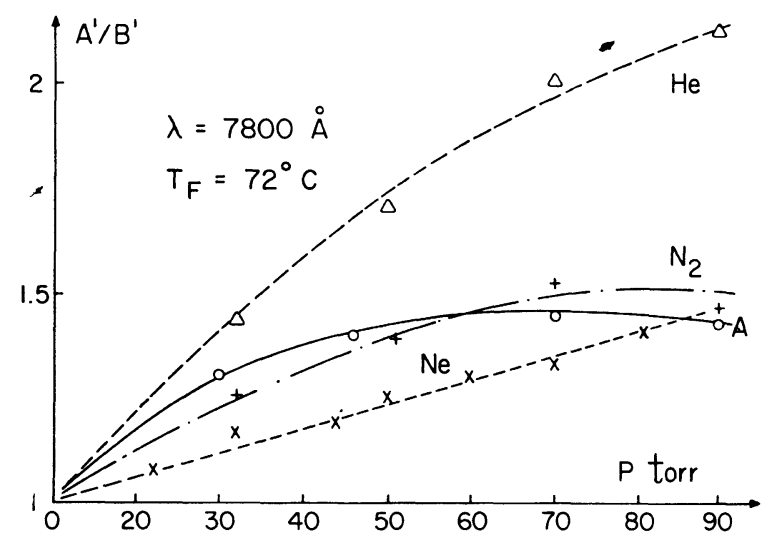

Fig. 5. - Même condition que dans la figure 4 sauf que les résultats donnés sont pour la raie $D_{2}$.

$\mathbf{A}^{\prime} / \mathbf{B}^{\prime}$ en fonction de la pression et de la nature du gaz tampon, avec une température de filtre de $72^{\circ} \mathrm{C}$. Le gaz qui donne le meilleur résultat est l'hélium. Les courbes des figures 6 et 7 nous donnent les résultats d'une série d'expériences avec ce gaz. La figure 8 nous montre les raies à deux stades du filtrage.

(2) Le rapport des raies est difficile à évaluer correctement à partir des résultats expérimentaux, à cause de la distorsion introduite par le filtrage dans la forme des raies : dans ce diagramme et tous ceux qui suivent nous avons exprimé ce rapport en fonction des maximums seulement, indépendamment des distorsions introduites.

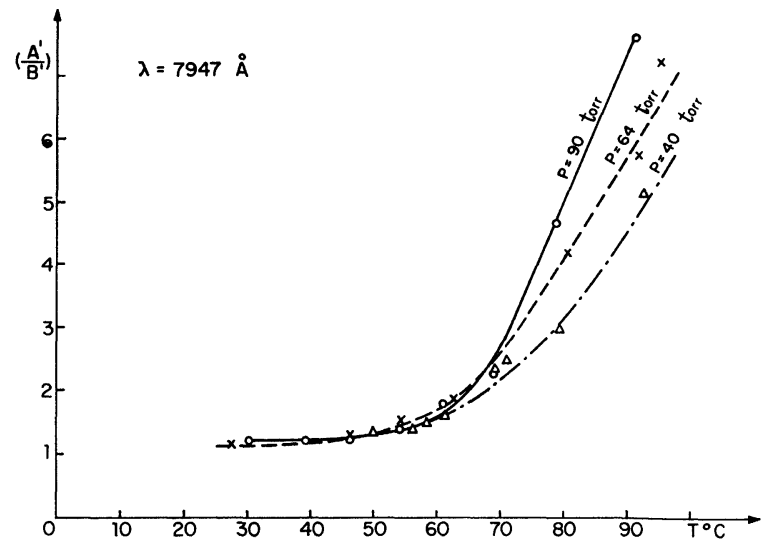

Fig. 6. - Efficacité du filtrage d'une lampe au rubidium 85 par un filtre isotopique contenant l'isotope rubidium 87 en présence d'hélium sous différentes pressions. Le paramètre en abscisse est la température du filtre. Les résultats donnés sont pour la raie $D_{1}$.

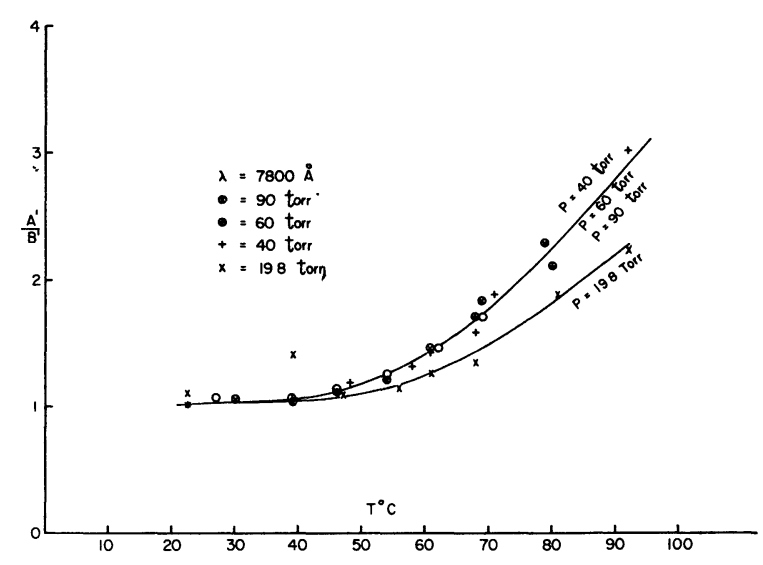

FIg. 7. - Même condition que dans la figure 6 sauf que les résultats sont donnés pour la raie $D_{2}$.

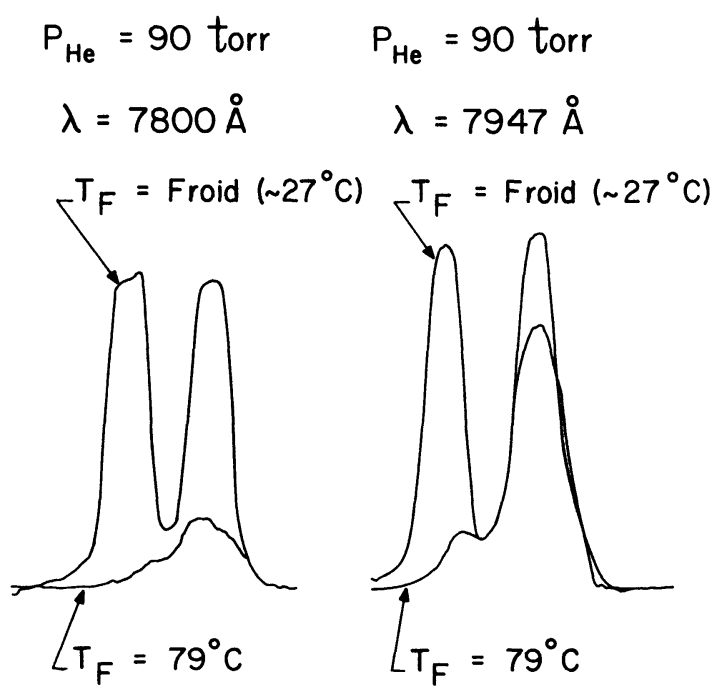

FIG. 8. - Forme des raies d'une lampe au rubidium 85 pour deux températures du filtre contenant du rubidium 87 . Le gaz tampon est de l'hélium. 
Une autre façon d'interpréter les résultats nous est donnée par les courbes de la figure 9. Soit $N_{1}$ et $N_{2}$ les populations des sous-niveaux 1 et 2 de l'état

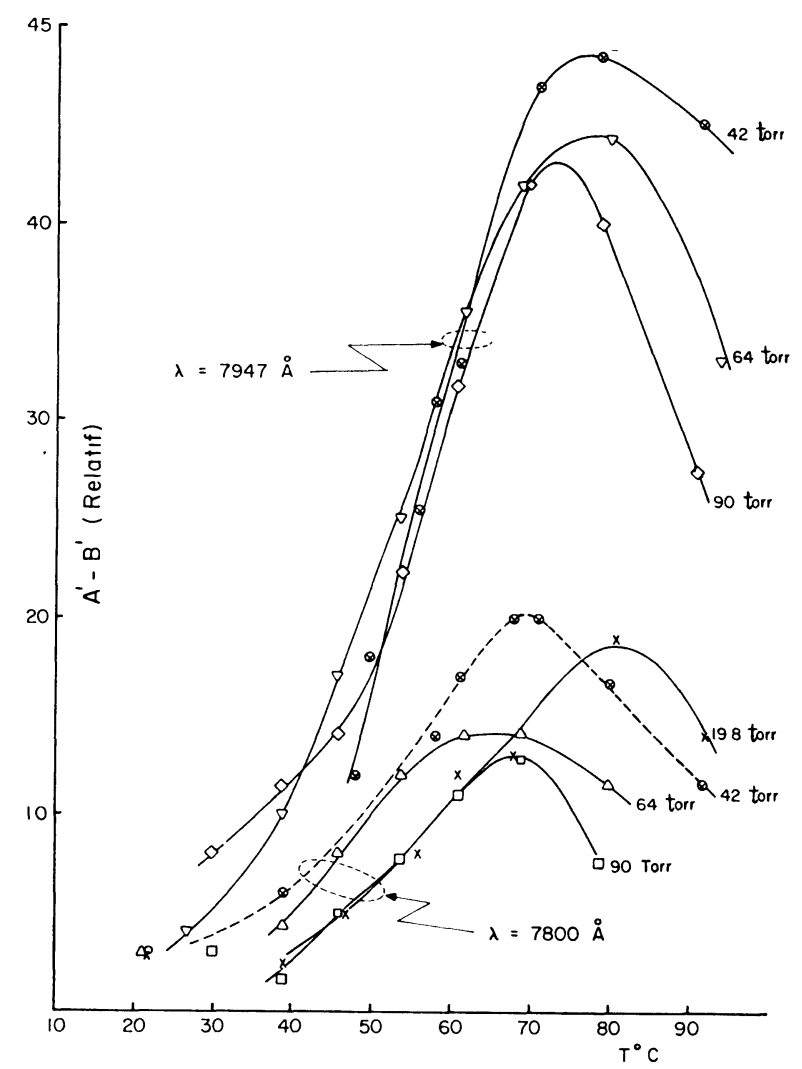

Fig. 9. - Influence de la température d'un filtre isotopique au rubidium 87 sur la différence d'intensité entre les composantes des doublets du spectre hyperfin d'une lampe au rubidium 85 . La pression du gaz tampon utilisé, soit l'hélium, apparaît comme paramètre. Le groupe des courbes inférieures correspond aux résultats obtenus pour la raie $D_{2}$. Les courbes du groupe supérieur sont pour la raie $\mathrm{D}_{1}$. fondamental; on peut alors écrire les relations suivantes :

$$
\begin{aligned}
\frac{\mathrm{d} N_{1}}{\mathrm{~d} t} & =\frac{k}{2}\left(N_{2} B^{\prime}-N_{1} A^{\prime}\right) \\
\frac{\mathrm{d} N_{2}}{\mathrm{~d} t} & =\frac{k}{2}\left(N_{1} A^{\prime}-N_{2} B^{\prime}\right)
\end{aligned}
$$

où $A^{\prime}$ et $B^{\prime}$ représentent les intensités des deux raies $\mathrm{A}^{\prime}$ et $\mathrm{B}^{\prime}$ filtrées, $k$ étant un coefficient représentant l'efficacité du pompage. Pour des faibles différences de population, on peut écrire :

$$
\frac{\mathrm{d} n}{\mathrm{~d} t}=\frac{k N}{2}\left(A^{\prime}-B^{\prime}\right)
$$

où $n=N_{2}-N_{1}$ et $N=N_{2}+N_{1}$. Si tout excès de population en 2 redescend en 1 en cédant son énergie par effet maser, le nombre maximum de photons radio-fréquence obtenu alors est proportionnel à $\left(A^{\prime}-B^{\prime}\right)$. Cette valeur peut représenter à un coefficient près, l'énergie maximum que l'on pourrait sortir d'un maser compte tenu des hypothèses précédentes.

Les courbes $A^{\prime}-B^{\prime}$ en fonction de la température sont tracées pour différentes pressions d'hélium. On voit qu'un maximum d'efficacité se situe vers 40 ou 50 torrs d'hélium avec une température de $70^{\circ} \mathrm{C}$. Remarquons que si l'on veut comparer les intensités de pompage pour les raies $D_{1}$ et $D_{2}$, il faut tenir compte de la différence de transmission des filtres, soit $50 \%$ et $75 \%$ respectivement. Les résultats décrits précédemment ont permis la réalisation d'un maser à rubidium 85 oscillant en régime permanent, en champ nul [17].

Remerciements. - Nous tenons à remercier M. JeanClaude Prince pour son aide qualifiée dans la construction des montages. Ces travaux ont été rendus possibles grâce à des subventions du Conseil National de Recherche, de la N. A. S. A., et du Conseil de Recherche pour la Défense. L'un des auteurs (G. M.) a bénéficié d'une bourse France-Québec.

\section{Bibliographie}

[1] Bender (P. L.), Beaty (E. C.) and Chi (A. R.), Phys. Rev. Letters, 1958, 1, 311.

[2] Davidovits (P.), Appl. Phys. Letters, 1964, 5, 15.

[3] Throne (D. H.), Proceedings of the 23rd Annual Symposium on Frequency Control, 274, 1969.

[4] Davidovits (P.) and Novick (R.), Proceedings of the IEEE, 1966, 54, 155.

[5] Vanier (J.), Phys. Rev., 1968, 168, 129.

[6] STERn (W. A.) and Novick (R.), Proceedings of the 23rd Annual Symposium on Frequency Control, 1969, 271.

[7] Hartmann (F.), J. Physique, 1967, 28, 288.

[8] Bederson (B.) and Jaccarino (V.), Phys. Rev., 1952, $87,228 \mathrm{~A}$

[9] Rabi (I. I.) and Senitzky (B.), Phys. Rev., 1956, 103, 315.
[10] Gibbs (H.), Thesis, Univ. of California, 1965 (Not published).

[11] Ch'en (S.) and Takeo (M.), Rev. Mod. Phys., 1957, 29, 20.

[12] Mitchell (A. C. G.) and Zemansky (M. W.), Resonance Radiation and excited atoms (Cambridge Univ. Press., New York) chap. 3, 1961.

[13] Alekseyev (E. I.) and Bazarov (N.), Radio Engineering and Electronic Physics, 1970, 15, 5.

[14] Barrat (J. P.) et Cohen-Tannoudji (C.), J. Physique Rad., 1961, 22, 329.

[15] Bazarov (N.) et GUBIN (V. P.), Radio Engineering and Electronic Physics, 1969, 14, 903.

[16] Chabbal (R.), Journal de Recherches du C. N. R. S., 1953, 24, 138.

[17] Vanier (J.), Vaillancourt (R.), Missout (G.), TÊtu (M.), J. Appl. Phys., 1970, 41, 3188. 